\section{Extracorporeal shock wave lithotripsy as a rescue for a trapped stone basket in the pancreatic duct}

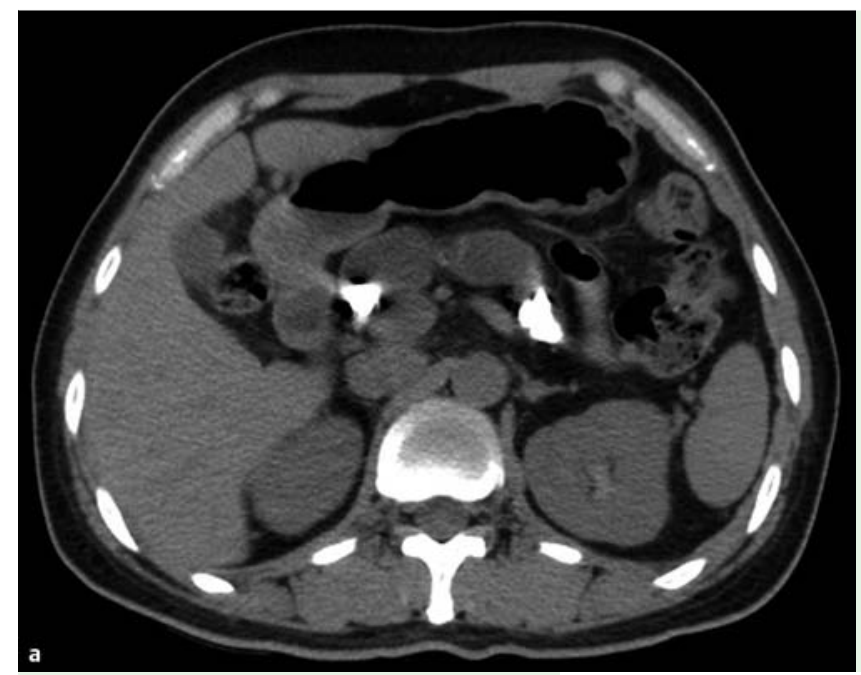

Fig. 1 A significantly dilated pancreatic duct with multiple radiopaque stones seen at: a computed tomography (CT); $\mathbf{b}$ magnetic resonance imaging (MRI).

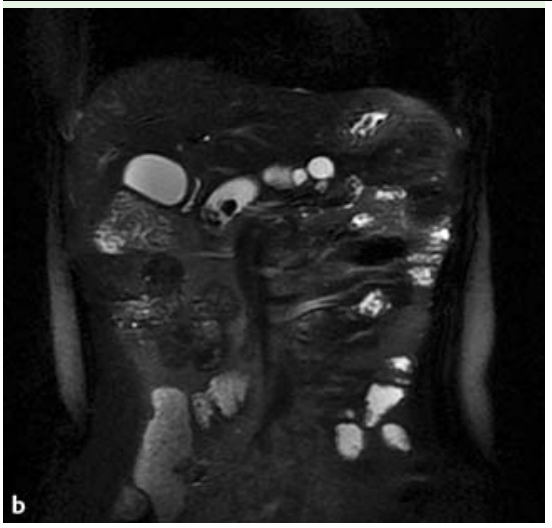

Video 1

The pancreatic orifice was widened to $0.8 \mathrm{~cm}$ via balloon sphincteroplasty at the minor papilla. The partly pulverized stones flowed out from the pancreatic orifice. Unfortunately, the residual stone was stuck near the pancreatic orifice at the papilla when we tried to remove it using a basket. Mechanical lithotripsy (through-thescope) also failed. We set the basket at its tight mode to fix the position of the residual stone near the pancreatic orifice at the papilla, cut the handle of the basket off, and then withdrew the duodenoscope.

\section{Video 2}

After ESWL, we attempted to drag out the basket directly, but considerable resistance was encountered. To avoid injury to the pancreatic duct or the gastrointestinal tract by forcible removal of the stone, we performed a second ERCP. We used a sphincterotome to disperse the pulverized stone in the basket and dragged it out. therapeutic session at an intensity of 6 $(16000 \mathrm{kV})$ on a scale of 1 to 6 , with a frequency of 120 shocks/min. Repeat sessions of ESWL were done on three successive days.

Most of the pancreatic stones were pulverized; however, a residual stone with a diameter of about $0.7 \mathrm{~cm}$ was left ( $\bullet$ Fig. 2 a). The location of the stone changed after hundreds of shocks and the targeting of the shock waves was repeatedly recalibrated. The stone was not significantly more pulverized with the fourth and fifth ESWL sessions ( $\bullet$ Fig. 2 b), even after we tried changing the angles of the generator and the position of the patient. Therefore, we decided to perform endoscopic retrograde cholangiopancreatography (ERCP) to remove the residual stone. After sphincterotomy, the pancreatic orifice was widened to $0.8 \mathrm{~cm}$ via balloon sphincteroplasty at the minor papilla. Unfortunately, the residual stone was stuck near the pancreatic orifice at the papilla when we tried to remove it using a basket. Mechanical lithotripsy (throughthe-scope) also failed.

We set the basket at its tight mode to fix the position of the residual stone near the pancreatic orifice at the papilla ( Fig.3a). We then cut off the handle of the basket, and withdrew the duodenoscope ( Video 1, and Fig.4). The patient was then immediately given her sixth ESWL treatment. The residual stone, now in a fixed position, could be accurately targeted and fragmented into smaller pieces ( $\bullet$ Fig. 3b).

After ESWL, we attempted to drag out the basket directly, but considerable resistance was encountered. Because of the fear that the stone fragments might injure the pancreatic duct or the gastrointestinal
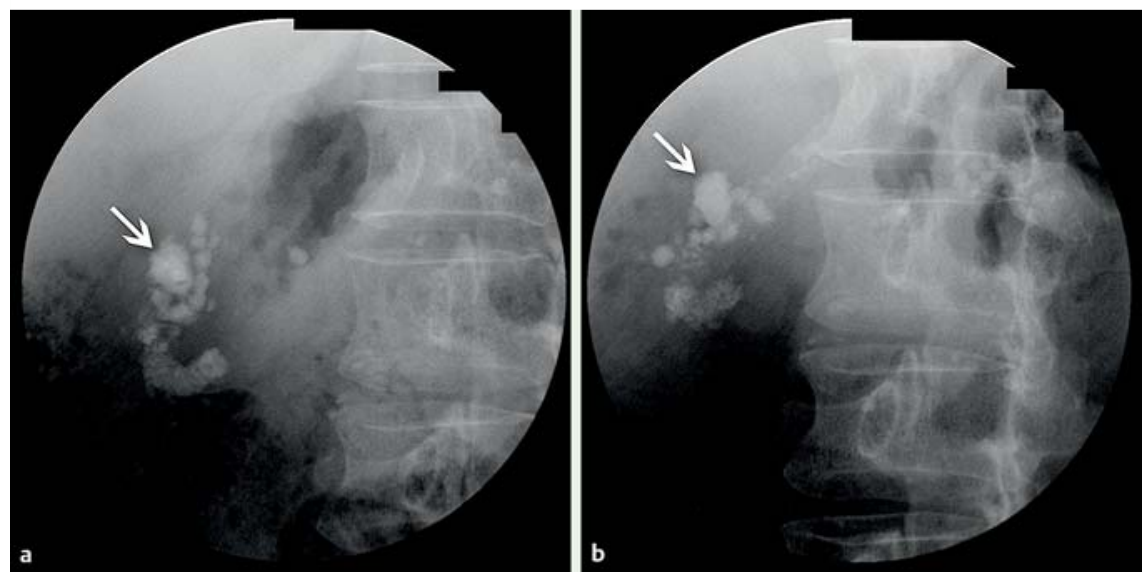

Fig. 2 a A residual stone (white arrow) with a diameter of about $0.7 \mathrm{~cm}$, that was hard to target after three sessions of endoscopic shock wave lithotripsy (ESWL). b The stone (white arrow) was not significantly pulverized after the fourth and fifth ESWL sessions. 

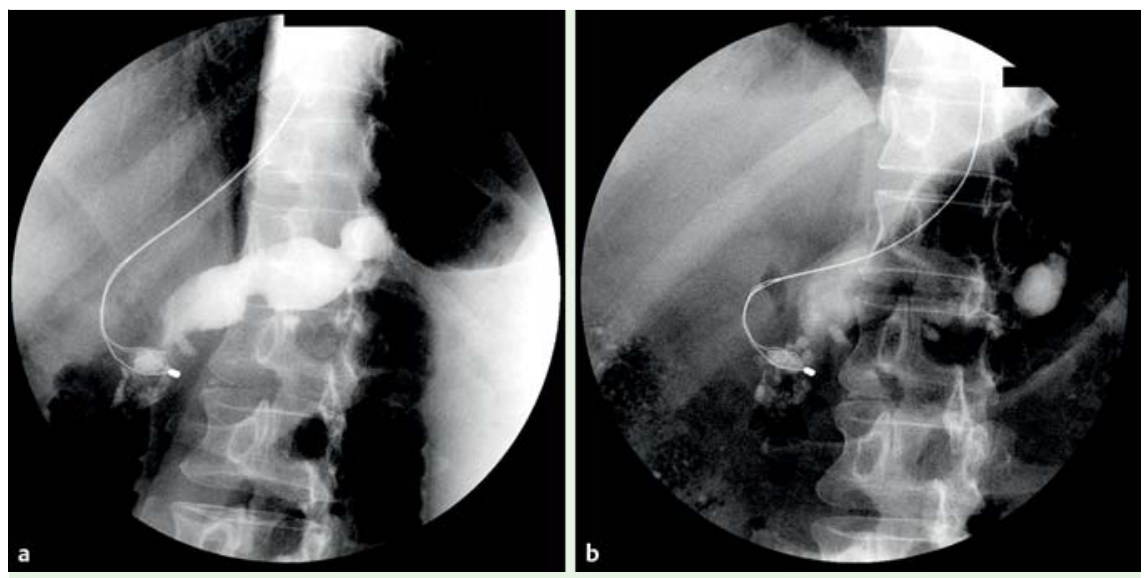

Fig. 3 a A basket was used to collect the residual stone, and was then tightened to fix the position of the residual stone near the pancreatic orifice at the papilla. $\mathbf{b}$ The stone was significantly pulverized after the sixth ESWL.

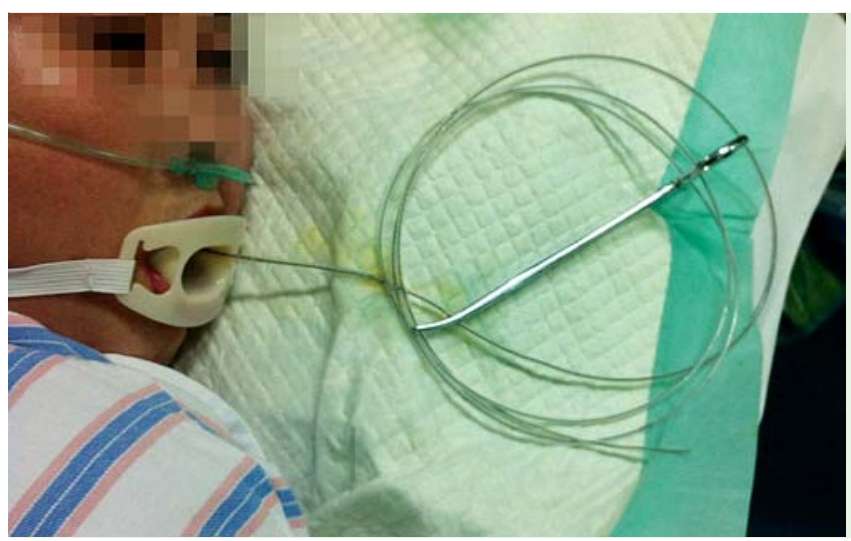

Fig. 4 The handle of the basket was cut off and the duodenoscope was withdrawn. The basket remained in the pancreatic duct, and the stub was clipped by a vascular clamp. tract if forcibly pulled out, we performed a second ERCP. We used a sphincterotome to disperse the pulverized stone in the basket and dragged it out ( Video 2 ).

Endoscopy_UCTN_Code_CPL_1AK_2AF

Competing interests: None

\section{Liang-Hao $\mathrm{Hu}^{1,}$ *, Ting-Ting $\mathrm{Du}^{1,}$ *, Zhuan Liao', *, Wen-Bin Zou², Bo Ye ${ }^{2}$, Zhao-Shen Li ${ }^{1}$}

${ }^{1}$ Department of Gastroenterology, Changhai Hospital, Second Military Medical University, Shanghai, China ${ }^{2}$ Digestive Endoscopy Center, Changhai Hospital, Second Military Medical University, Shanghai, China

\section{Bibliography}

DOI http://dx.doi.org/

10.1055/s-0034-1377221

Endoscopy 2014; 46: E332-E333

(c) Georg Thieme Verlag KG

Stuttgart · New York

ISSN 0013-726X

Corresponding author

Zhao-Shen Li, MD

Department of Gastroenterology

Changhai Hospital

Second Military Medical University

168 Chang-hai Road

Shanghai 200433

China

Fax: +86-21-55620081

zhaoshen-li@hotmail.com

\footnotetext{
* Liang-Hao Hu, Ting-Ting Du, and Zhuan Liao contributed equally to this work.
} 